\section{AR-V7 status in CTCs is a treatment-specific biomarker}

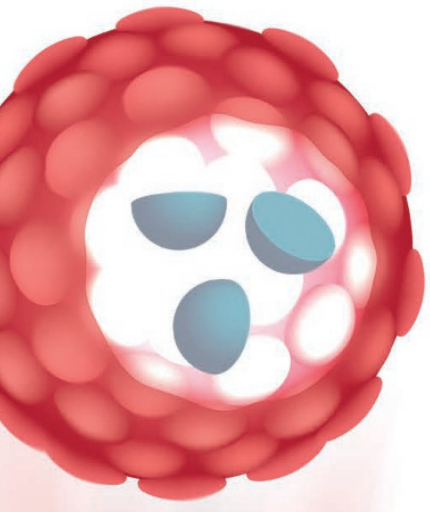

Pretherapy nuclear expression of androgen receptor splice variant 7 (AR-V7) in circulating tumour cells (CTCs) is a treatment-specific biomarker in men with metastatic castration-resistant prostate cancer (mCRPC), according to new data reported in JAMA Oncology. Expression of this androgen receptor variant in CTCs was associated with superior responses to taxanes but inferior responses to androgenreceptor-signalling (ARS) inhibitors.

Howard Scher and colleagues conducted this cross-sectional study involving 161 patients with progressive $\mathrm{mCRPC}$ who received 193 treatments at Memorial Sloan Kettering Cancer Center between 2012 and 2013. In total, $80.8 \%$ of patients had a single therapy, $18.6 \%$ received two therapies and $0.6 \%$ underwent three therapies. Blood was collected from men immediately before they started a new line of systemic therapy and, overall, 191 blood samples were evaluable, 128 before an ARS inhibitor and 63 before taxane therapy. The main outcomes were serum PSA response, time receiving therapy, radiographic progression-free survival (rPFS) and overall survival.

Analysis of AR-V7 expression by immunofluorescent staining revealed that it is expressed in a variety of CTCs including cytokeratin-positive single CTCs and CTC clusters, and cytokeratin-negative CTCs. Most CTCs were AR-V7-negative, but

...overall

survival was

longer for men

with AR-V7-

positive CTCs

who received a

taxane...

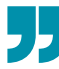

AR-V7-positive in men who had previous first-line therapy, $18 \%$ being positive in those who had received second-line therapy and $31 \%$ testing positive in men who had undergone third-line therapy.

Of the patients who went on to receive ARS inhibitors, 37\% had sensitive post-therapy PSA changes (defined as $\geq 50 \%$ decline from baseline levels at 12 weeks) and $63 \%$ had resistant changes (failure to reach a $50 \%$ decrease in PSA concentration). None of the patients who experienced PSA sensitivity to ARS inhibition had AR-V7-posistive CTCs, whereas $20 \%$ of men who had a resistant response did have AR-V7-positive CTCs before therapy. Subset analysis showed that overall survival was significantly worse for patients with pretherapy AR-V7-positive CTCs $(P<0.001)$. AR-V7-positive status before ARS inhibitor therapy was also associated with worse outcomes regarding $\mathrm{rPFS}$ and time on therapy.

AR-V7-positive CTC status did not affect outcomes for rPFS or time on therapy for those men who went on to receive a taxane; however, overall survival was significantly increased for men who were negative for AR-V7 expression in their CTCs. Relatively, median overall survival was longer for men with AR-V7-positive CTCs who received a taxane than for those who received an ARS inhibitor (8.9 versus 4.6 months). AR-V7 status remained the most significant factor influencing outcomes in a multivariate model, showing that patients with AR-V7-positive CTCs had more favourable survival times with taxanes than with ARS inhibitors, whereas no such difference was observed for men with AR-V7-negative CTCs.

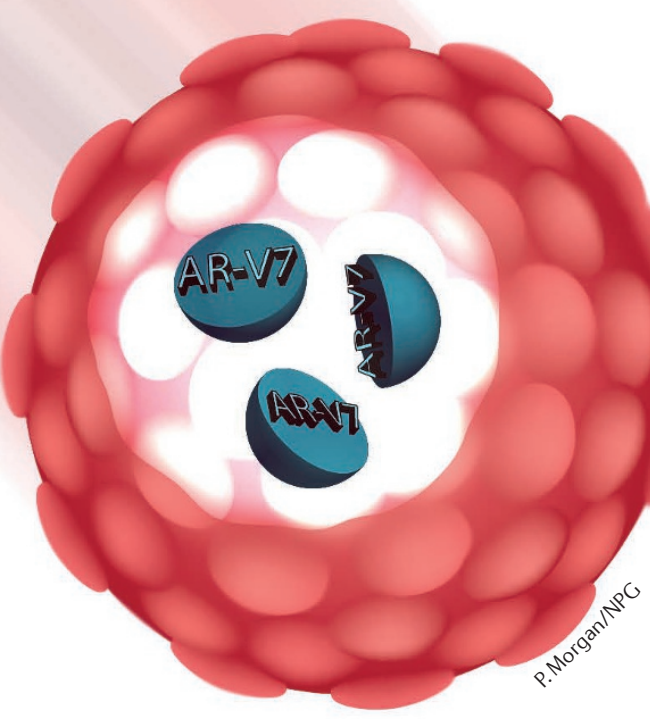

These data indicate that the pretherapy AR-V7 status of CTCs in men with $\mathrm{MCRPC}$ could be a treatment-specific biomarker, with the clinical implication being that if AR-V7 is detected in CTCs then taxane therapy, not an ARS inhibitor, should be chosen as the next course of treatment. In an accompanying commentary, Montgomery and Plymate state "...the ability of the assay to direct patients away from ineffective therapy has the greatest potential for benefit." They continue “...AR-V7 protein in CTCs will provide an extremely useful positive response biomarker for taxane treatment." Noting that "the clear implication is that if AR splice variants are truly driving tumour biology, then AR-V7 and other AR splice variants should be considered as relevant therapeutic targets."

Louise Stone

ORIGINAL ARTICLE Scher, H. I. et al. Association of AR-V7 on circulating tumor cells as a treatmentspecific biomarker with outcomes and survival in castration-resistant prostate cancer. JAMA Oncol. http://dx.doi.org/10.1001/jamaoncol.2016.1828 (2016)

FURTHER READING Montgomery, R. B. \& Plymate, S. R. AR-V7 protein in circulating tumor cells-the decider for therapy? JAMA Oncol. http://dx.doi.org/10.1001/jamaoncol.2016.2360 (2016) 